TRANSACTIONS OF THE

AMERICAN MATHEMATICAL SOCIETY

Volume 353, Number 2, Pages 539-556

S 0002-9947(00)02371-0

Article electronically published on October 13, 2000

\title{
AN ARITHMETIC PROPERTY OF FOURIER COEFFICIENTS OF SINGULAR MODULAR FORMS ON THE EXCEPTIONAL DOMAIN
}

\author{
SHOU-TE CHANG AND MINKING EIE
}

\begin{abstract}
We shall develop the theory of Jacobi forms of degree two over Cayley numbers and use it to construct a singular modular form of weight 4 on the 27-dimensional exceptional domain. Such a singular modular form was obtained by Kim through the analytic continuation of a nonholomorphic Eisenstein series. By applying the results in a joint work with Eie, A. Krieg provided an alternative proof that a function with a Fourier expansion obtained by Kim is indeed a modular form of weight 4 . This work provides a systematic and general approach to deal with the whole issue.
\end{abstract}

\section{INTRODUCTION}

Let $\mathbf{H}$ be the upper half-plane and let $\mathbf{C}$ be the field of complex numbers. The theory of Jacobi forms on $\mathbf{H} \times \mathbf{C}, \mathbf{H} \times \mathbf{C}^{2}$ and $\mathbf{H} \times \mathbf{C}^{4}$ was substantially investigated for various purposes. The theory of Jacobi forms on $\mathbf{H} \times \mathbf{C}^{8}$ (or over Cayley numbers) was initiated by Eie (3]) and A. Krieg $([4,5])$ in 1991 to investigate modular forms which lie in the Maass space on the upper half-plane of $2 \times 2$ Hermitian matrices over Cayley numbers. Its close connection with the 27-dimensional exceptional domain makes the subject especially interesting. How to construct a theta series on the exceptional domain is still an open problem. However, a theta series on its 10-dimensional boundary, the upper half-plane of $2 \times 2$ Hermitian matrices over Cayley numbers, was constructed by Eie and A. Krieg (see [4) as an example of singular modular form of weight 4, which was later also obtained by Kim in 1993. Kim followed the method proposed by Shimura to obtain a singular modular form of weight 4 on the exceptional domain. In particular, Kim was able to determine the Fourier coefficients of such a singular modular form. In 1996, A. Krieg (see [9]) provided an alternative proof that a function with such a Fourier expansion is indeed a modular form of weight 4 by using the theory of Jacobi forms over Cayley numbers developed by Eie and Krieg (see [4, 5]).

In this paper, we provide a detailed treatment of this approach. We shall prove two identities involving Fourier coefficients of $E_{4,0}(Z)$, the singular modular form of weight 4 obtained by Kim, and use these coefficients to construct Jacobi forms of weight 4 and index $m$ on $\mathcal{H}_{2} \times \mathcal{C}_{\mathbf{C}}^{2}$. These Jacobi forms provide the coefficients

Received by the editors March 14, 1997 and, in revised form, October 28, 1997, January 27, 1998, and April 1, 1998.

1991 Mathematics Subject Classification. Primary 11F55, 11F72.

This work was supported by the Department of Mathematics, National Chung Cheng University, and by the National Science Foundation of Taiwan, Republic of China. 
of the Fourier-Jacobi expansion of a modular form on the exceptional domain. Consequently, we obtain a modular form of weight 4 if we begin with these identities. This provides an alternative way to construct a singular modular form on the exceptional domain via the theory of Jacobi forms on $\mathcal{H}_{2} \times \mathcal{C}_{\mathbf{C}}^{2}$, which we develop in Section 5.

Here we outline the basic ideas behind our construction. Note that the Fourier coefficients of singular modular forms are wholly determined by their images under Siegel's $\Phi$-operator (see [8]). On the other hand, the $m$-th coefficient of the FourierJacobi expansion of a modular form of weight $k$ on the exceptional domain must be a Jacobi form of weight $k$ and index $m$, and hence it corresponds to a vector-valued modular form of weight $k-4$. The corresponding vector-valued modular form of a singular modular form of weight 4 is nothing but a constant vector: an eigenvector of a certain unitary matrix of size $m^{16} \times m^{16}$. Of course, we are able to construct such a constant vector from its images under Siegel's $\Phi$-operator.

Hence our construction of singular modular forms is clear. First we construct a constant vector of size $m^{16} \times 1$ from the Fourier coefficients of $f_{4}(Z)$ given in [4]. We prove that such a vector is indeed an eigenvector of the unitary matrix $A \otimes A$, where $A=\frac{1}{m^{4}}\left[e^{2 \pi i \sigma(p, q) / m}\right]_{p, q: \boldsymbol{O} / m \boldsymbol{O}}$. Thus, it corresponds to a Jacobi form of weight 4 and index $m$. This is precisely the $m$-th coefficient in the Fourier-Jacobi expansion of the singular modular form of weight 4 we need.

\section{NotATiOn}

As usual $\mathbf{Z}, \mathbf{Q}, \mathbf{R}, \mathbf{C}$ denote the ring of integers, the field of rational numbers, real numbers and complex numbers respectively. For any field $f, \mathcal{C}_{f}$ is an eightdimensional vector space over $f$ with a basis $\left\{e_{j}: j=0,1,2, \ldots, 7\right\}$ which is characterized by the following rules for multiplication (see [1]):

(1) $x e_{0}=e_{0} x=x$ for all $x \in \mathcal{C}_{f}$;

(2) $e_{j}^{2}=-e_{0}$ for $j=1,2, \ldots, 7$; and

(3) $e_{1} e_{2} e_{4}=e_{2} e_{3} e_{5}=e_{3} e_{4} e_{6}=e_{4} e_{5} e_{7}=e_{5} e_{6} e_{1}=e_{6} e_{7} e_{2}=e_{7} e_{1} e_{3}=-e_{0}$.

For $x=\sum_{j=0}^{7} x_{j} e_{j} \in \mathcal{C}_{f}$, we let

(i) $\bar{x}=x_{0} e_{0}-\sum_{j=1}^{7} x_{j} e_{j}$;

(ii) $N(x)=x \bar{x}=\sum_{j=0}^{7} x_{j}^{2}$;

(iii) $T(x)=2 x_{0}=x+\bar{x}$; and

(iv) $\sigma(x, y)=2 \sum_{j=0}^{7} x_{j} y_{j}$ if $y=\sum_{j=0}^{7} y_{j} e_{j} \in \mathcal{C}_{f}$.

Note that

$$
N(x+y)=N(x)+N(y)+\sigma(x, y)
$$

and

$$
\sigma(x, y)=T(\bar{x} y)=T(x \bar{y}) .
$$


Let $\boldsymbol{o}$ be the ring of integral Cayley numbers. Then $\boldsymbol{o}$ is the $\mathbf{Z}$-module in $\mathcal{C}_{\mathbf{Q}}$ generated by the following elements:

$$
\begin{gathered}
\alpha_{0}=e_{0}, \quad \alpha_{1}=e_{1}, \quad \alpha_{2}=e_{2}, \quad \alpha_{3}=-e_{4}, \\
\alpha_{4}=\frac{1}{2}\left(e_{1}+e_{2}+e_{3}-e_{4}\right), \quad \alpha_{5}=\frac{1}{2}\left(-e_{0}-e_{1}-e_{4}+e_{5}\right), \\
\alpha_{6}=\frac{1}{2}\left(-e_{0}+e_{1}-e_{2}+e_{6}\right), \quad \alpha_{7}=\frac{1}{2}\left(-e_{0}+e_{2}+e_{4}+e_{7}\right) .
\end{gathered}
$$

Indeed $\boldsymbol{o}$ is characterized as the maximal $\mathbf{Z}$-module in $\mathcal{C}_{\mathbf{Q}}$ with the following properties:

(a) $T(x) \in \mathbf{Z}$ and $N(x) \in \mathbf{Z}$ for all $x$ in the set;

(b) the set is closed under subtraction and multiplication; and

(c) the set contains 1 .

Let $\mathcal{J}=\mathcal{J}_{\mathbf{R}}$ be the set of $3 \times 3$ Hermitian matrices over Cayley numbers. Then $\mathcal{J}$ consists of matrices of the form

$$
\left[\begin{array}{ccc}
\xi_{1} & x_{12} & x_{13} \\
\bar{x}_{12} & \xi_{2} & x_{23} \\
\bar{x}_{13} & \bar{x}_{23} & \xi_{3}
\end{array}\right] \text {, where } \xi_{1}, \xi_{2}, \xi_{3} \in \mathbf{R} \text { and } x_{12}, x_{13}, x_{23} \in \mathcal{C}_{\mathbf{R}}
$$

Let $\mathcal{J}_{\boldsymbol{o}}=\mathcal{J} \cap M_{3}(\boldsymbol{o})$. For $1 \leq i, j \leq 3$, let $e_{i j}$ be the $3 \times 3$ matrix with 1 at the (ij)-position and 0 elsewhere. When $i \neq j$ and $t \in \mathcal{C}_{\mathbf{R}}$, we let $U_{i j}(t)=E+t e_{i j}$, with $E$ being the $3 \times 3$ identity matrix.

We supply $\mathcal{J}$ with a product defined by

$$
X \circ Y=\frac{1}{2}(X Y+Y X)
$$

where $X Y$ is the ordinary matrix product. Then $\mathcal{J}$ becomes a real Jordan algebra with this product. For $X \in \mathcal{J}$ as given in (2.1), we define

(1) $\operatorname{tr}(X)=\xi_{1}+\xi_{2}+\xi_{3}$;

(2) $\operatorname{det} X=\xi_{1} \xi_{2} \xi_{3}-\xi_{1} N\left(x_{23}\right)-\xi_{2} N\left(x_{13}\right)-\xi_{3} N\left(x_{12}\right)+T\left(\left(x_{12} x_{23}\right) \bar{x}_{13}\right) ;$ and

(3) $X \times X=X^{2}-\operatorname{tr}(X) X+\frac{1}{2}\left(\operatorname{tr}(X)^{2}-\operatorname{tr}\left(X^{2}\right)\right) E$

$$
=\left[\begin{array}{ccc}
\xi_{2} \xi_{3}-N\left(x_{23}\right) & x_{13} \bar{x}_{23}-\xi_{3} x_{12} & x_{12} x_{23}-\xi_{2} x_{13} \\
x_{23} \bar{x}_{13}-\xi_{3} \bar{x}_{12} & \xi_{1} \xi_{3}-N\left(x_{13}\right) & \bar{x}_{12} x_{13}-\xi_{1} x_{23} \\
\bar{x}_{23} \bar{x}_{13}-\xi_{2} \bar{x}_{13} & \bar{x}_{13} x_{12}-\xi_{1} \bar{x}_{23} & \xi_{1} \xi_{2}-N\left(x_{12}\right)
\end{array}\right] .
$$

Note that $X$ is invertible if and only if $\operatorname{det} X \neq 0$, and in this case the inverse is given by

We define

$$
X^{-1}=\frac{1}{\operatorname{det} X}(X \times X) \text {. }
$$

$\operatorname{rank} X=1$ iff $X \neq 0$ and $X \times X=0$ $\operatorname{rank} X=2$ iff $X \times X \neq 0$ and $\operatorname{det} X=0$, $\operatorname{rank} X=3$ iff $\operatorname{det} X \neq 0$.

We will also define an inner product on $\mathcal{J}$ by setting

$$
(X, Y)=\operatorname{tr}(X \circ Y) \text {. }
$$


Finally, we let $\mathcal{K}$ be the set of squares $X \circ X$ of elements of $\mathcal{J}$ and $\mathcal{K}^{+}$the interior of $\mathcal{K}$.

The exceptional domain contained in $\mathbf{C}^{27}$ is thus defined by

$$
\mathcal{H}=\left\{Z=X+i Y \mid X, Y \in \mathcal{J}_{\mathbf{R}}, Y \in \mathcal{K}^{+}\right\} .
$$

The exceptional domain $\mathcal{H}$ is a tube domain on which Baily started the study of automorphic forms in 1970. He introduced the Eisenstein series and proved that it has a Fourier expansion with rational Fourier coefficients given by Euler products. The group of holomorphic automorphisms $\mathcal{G}$ of $\mathcal{H}$ is a Lie group of type $E_{7}$ (see [1]). Let $\Gamma$ be the discrete subgroup of $\mathcal{G}_{\mathbf{R}}$ generated by the following automorphisms of $\mathcal{H}$ :

(i) $\iota: Z \longrightarrow-Z^{-1}$,

(ii) $p_{B}: Z \longrightarrow Z+B$ for $B \in \mathcal{J}_{\boldsymbol{o}}$, and

(iii) $t_{U}: Z \longrightarrow Z[U]={ }^{t} \bar{U} Z U$, where $U=U_{i j}(t)$ for $t \in \boldsymbol{o}$.

Let $k$ be an integer. A holomorphic function $f$ defined on $\mathcal{H}$ is a modular form of weight $k$ with respect to $\Gamma$ if it satisfies the following conditions:

(a) $f\left(-Z^{-1}\right)=(\operatorname{det}(-Z))^{k} f(Z)$; and

(b) $f(Z[U]+B)=f(Z)$ where $B \in \mathcal{J}_{\boldsymbol{o}}$ and $U=U_{i j}(t)$ for $t \in \boldsymbol{o}$.

In particular, from (b) above, a modular form $f$ on $\mathcal{H}$ has a Fourier expansion of the form

$$
f(Z)=\sum_{T \in \mathcal{K} \cap \mathcal{J}_{\boldsymbol{O}}} a(T) e^{2 \pi i(T, Z)},
$$

and furthermore, $f$ is a singular modular form if $a(T)=0$ unless $\operatorname{det} T=0$.

\section{A Singular MOdUlar FORM OF WEIGHT 4}

Consider the upper half-plane of Hermitian matrices over Cayley numbers defined by

$$
\mathcal{H}_{2}=\left\{Z=X+i Y \mid{ }^{t} \bar{X}=X \in M_{2}\left(\mathcal{C}_{\mathbf{R}}\right),{ }^{t} \bar{Y}=Y \in M_{2}\left(\mathcal{C}_{\mathbf{R}}\right), Y>0\right\} .
$$

The half-plane $\mathcal{H}_{2}$ corresponds to a proper boundary component of the exceptional domain $\mathcal{H}$. It was first investigated in $[3$ in order to construct modular forms in the Maass space. A holomorphic function $f: \mathcal{H}_{2} \longrightarrow \mathbf{C}$ is called a modular form of weight $k$ if $f$ satisfies the following conditions:

(1) $f(Z+B)=f(Z)$ for all $B=\left[\begin{array}{cc}n & t \\ \bar{t} & m\end{array}\right]$, where $n, m \in \mathbf{Z}$ and $t \in \boldsymbol{o}$;

(2) $f(Z[U])=f(Z)$ for all $U=\left[\begin{array}{ll}0 & 1 \\ 1 & 0\end{array}\right],\left[\begin{array}{ll}1 & t \\ 0 & 1\end{array}\right]$ or $\left[\begin{array}{ll}1 & 0 \\ t & 1\end{array}\right]$, where $t \in \boldsymbol{o}$; and

(3) $f\left(-Z^{-1}\right)=(\operatorname{det} Z)^{k} f(Z)$.

In particular, $f$ has a Fourier expansion of the form

$$
\sum_{T \geq 0} a(T) e^{2 \pi i(T, Z)}
$$

if $f$ is a modular form of weight $k$. Here $(T, Z)=t_{1} z+t_{2} z^{*}+\sigma\left(t_{12}, w\right)$ if

$$
T=\left[\begin{array}{cc}
t_{1} & t_{12} \\
\bar{t}_{12} & t_{2}
\end{array}\right] \text { and } Z=\left[\begin{array}{cc}
z & w \\
\bar{w} & z^{*}
\end{array}\right] .
$$


A modular form $f$ is a singular modular form if $a(T)=0$, unless rank $T=0$ or 1 . The following was given in [4] as an example of a singular modular form of weight 4.

Proposition 1 ([4). The theta series

$$
f_{4}(Z)=\sum_{h \in \boldsymbol{O}^{2}} e^{2 \pi i\left(h^{t} \bar{h}, Z\right)}, \text { where } Z \in \mathcal{H}_{2},
$$

is a singular modular form of weight 4 with its Fourier coefficients given by

$$
a\left(\left[\begin{array}{cc}
N(t) / m & t \\
t & m
\end{array}\right]\right)=240 \sum_{d \mid(m, t, N(t) / m)} d^{3}
$$

where $d \mid(m, t, N(t) / m)$ stands for $d^{-1} m \in \mathbf{Z}, d^{-1} t \in \boldsymbol{o}$ and $d^{-1}(N(t) / m) \in \mathbf{Z}$.

For each positive integer $m$ and integral Cayley number $q$, we define

$$
F_{m}(q)=\left\{\begin{array}{cl}
240 \sum_{d \mid(m, t, N(q) / m)} d^{3}, & \text { if } N(q) \equiv 0 \quad(\bmod m), \\
0, & \text { otherwise. }
\end{array}\right.
$$

Then the Fourier-Jacobi expansion of $f_{4}(Z)$ is given by

$$
f_{4}(Z)=\sum_{t \in \boldsymbol{O}} e^{2 \pi i N(t) z}+\sum_{m=1}^{\infty} \varphi_{m}(z, w) e^{2 \pi i m z^{*}},
$$

where

$$
Z=\left[\begin{array}{cc}
z & w \\
\bar{w} & z^{*}
\end{array}\right]
$$

and

$$
\varphi_{m}(z, w)=\sum_{q: \boldsymbol{O} / m \boldsymbol{O}} F_{m}(q) \sum_{\lambda \in \boldsymbol{O}} \exp \left\{2 \pi i m\left[N\left(\lambda+\frac{q}{m}\right) z+\sigma\left(\lambda+\frac{q}{m}, w\right)\right]\right\} .
$$

Set

$$
\vartheta_{m, q}(z, w)=\sum_{\lambda \in \boldsymbol{O}} \exp \left\{2 \pi i m\left[N\left(\lambda+\frac{q}{m}\right) z+\sigma\left(\lambda+\frac{q}{m}, w\right)\right]\right\} .
$$

The following properties can be verified directly from the definition above:

(1) $\vartheta_{m, q}(z+1, w)=e^{2 \pi i N(q) / m} \vartheta_{m, q}(z, w)$;

(2) $\vartheta_{m, q_{1}}(z, w)=\vartheta_{m, q_{2}}(z, w)$ if $q_{1} \equiv q_{2}(\bmod m)$; and

(3) $\vartheta_{m, q}(z,-w)=\vartheta_{m,-q}(z, w)$.

With the help of the Poisson Summation Formula or employing the result in 4 . Proposition 1] we also have

(4) $\vartheta_{m, q}\left(-\frac{1}{z}, \frac{w}{z}\right)=z^{4} e^{2 \pi i N(w) / z} \cdot \frac{1}{m^{4}} \sum_{p \in \boldsymbol{O} / m \boldsymbol{O}} e^{-2 \pi i \sigma(q, p) / m} \vartheta_{m, q}(z, w)$.

Following the argument of [5, Theorem 1], this can lead to the identity

$$
F_{m}(q)=\frac{1}{m^{4}} \sum_{p \in \boldsymbol{O} / m \boldsymbol{O}} e^{2 \pi i \sigma(q, p) / m} F_{m}(p) .
$$

Let $\varphi$ be the group homomorphism from $S L_{2}(\mathbf{Z})$ to $U\left(m^{8}\right)$, the unitary group of size $m^{8} \times m^{8}$, determined by

(a) $\varphi\left(\left[\begin{array}{ll}1 & 1 \\ 0 & 1\end{array}\right]\right)=\operatorname{diag}\left[e^{2 \pi i N\left(q_{1}\right) / m}, \ldots, e^{2 \pi i N\left(q_{m} 8\right) / m}\right]$, and 
(b) $\varphi\left(\left[\begin{array}{cc}0 & -1 \\ 1 & 0\end{array}\right]\right)=\frac{1}{m^{4}}\left[e^{2 \pi i \sigma\left(q_{\mu}, q_{\nu}\right) / m}\right]_{1 \leq \mu, \nu \leq m^{8}}$.

Here $q_{1}, q_{2}, \ldots, q_{m^{8}}$ is a set of representatives of $\boldsymbol{o} / m \boldsymbol{o}$. Set

$$
F_{m}={ }^{t}\left[F_{m}\left(q_{1}\right), \ldots, F_{m}\left(q_{m^{8}}\right)\right] .
$$

Then

$$
F_{m}=\varphi\left(\left[\begin{array}{ll}
1 & 1 \\
0 & 1
\end{array}\right]\right) F_{m}
$$

and

$$
F_{m}=\varphi\left(\left[\begin{array}{rr}
0 & -1 \\
1 & 0
\end{array}\right]\right) F_{m}
$$

Although identity (I) can be extracted from [4, 5] as shown above, we will give a detailed alternative proof in the next section because of its elegant arithmetic properties. Note that since $F_{m}(q)$ is a multiplicative function of $m$, it suffices to prove the cases when $m=l^{\nu}$ for some prime number $l$ and nonnegative integer $\nu$.

\section{THE $p$-ADIC VERSION OF THE FIRST IDENTITY}

In this section, we shall provide a purely arithmetic proof that the vector $F_{m}=$ ${ }^{t}\left(F_{m}(q)\right)_{q \in \boldsymbol{O} / m \boldsymbol{O}}$ is indeed an eigenvector of the matrix

$$
A=\frac{1}{m^{4}}\left(e^{2 \pi i \sigma(q, p) / m}\right)_{q, p \in \boldsymbol{O} / m \boldsymbol{O}} .
$$

Or equivalently, one has identity (I)

$$
F_{m}(q)=\frac{1}{m^{4}} \sum_{p: \boldsymbol{O} / m \boldsymbol{O}} e^{2 \pi i \sigma(q, p) / m} F_{m}(p) .
$$

Fix a prime number $l$ and a nonnegative integer $\nu$. For any integral Cayley number $q$ with $N(q) \equiv 0\left(\bmod l^{\nu}\right)$, define

$$
\begin{aligned}
\tau(q) & =\tau\left(l^{\nu}, q\right) \\
& =\max \left\{k \in \mathbf{Z} \mid 0 \leq k \leq \nu, l^{-k} q \in \boldsymbol{o} / l^{\nu} \boldsymbol{o}, l^{-(k+\nu)} N(q) \in \mathbf{Z}\right\} .
\end{aligned}
$$

Then we can rewrite $F_{\nu}(q) \equiv F_{l^{\nu}}(q)$ as

$$
F_{\nu}(q)=\left\{\begin{array}{cl}
240 \sum_{k=0}^{\tau\left(l^{\nu}, q\right)} l^{3 k}, & \text { if } N(q) \equiv 0 \quad\left(\bmod l^{\nu}\right), \\
0, & \text { otherwise. }
\end{array}\right.
$$

We first prove the case $q=0$.

Proposition 1. Let $l$ be a prime number and $\nu$ a nonnegative integer. Suppose that $F_{\nu}(q)$ is defined as in (4.2). Then

$$
\frac{1}{240} \cdot l^{-4 \nu} \sum_{p: \boldsymbol{O} / l^{\nu} \boldsymbol{O}} F_{\nu}(p)=\sum_{k=0}^{\nu} l^{3 k}=\frac{1}{240} \cdot F_{\nu}(0) .
$$


Proof. For any nonnegative integer $j$ and an integral Cayley number $p$, let

$$
T_{j}(p)=\left[\begin{array}{cc}
l^{-j} N(p) & p \\
\bar{p} & l^{j}
\end{array}\right] .
$$

For each integer $j$ with $0 \leq j \leq \nu$, set

$$
R_{j}=\left\{T_{j}(p) \mid p \in \boldsymbol{o} / l^{j} \boldsymbol{o}, N(p) \equiv 0 \quad\left(\bmod l^{j}\right)\right\} .
$$

Note that if $T_{j}(p) \in R_{j}$ and $\tau(p) \geq 1$, then $l^{-1} T_{j}(p) \in R_{j-1}$. By our definition of $F_{\nu}(p)$, we then have

$$
\begin{aligned}
\frac{1}{240} & \cdot l^{-4 \nu} \sum_{p: \boldsymbol{O} / l^{\nu} \boldsymbol{O}} F_{\nu}(p) \\
& =l^{-4 \nu}\left|R_{\nu}\right|+l^{-4 \nu} \cdot l^{3} \cdot\left|R_{\nu-1}\right|+\ldots+l^{-4 \nu} l^{3(\nu-1)}\left(R_{1}\right)+l^{-\nu},
\end{aligned}
$$

where $\left|R_{j}\right|$ denotes the number of matrices in $R_{j}$.

By the Corollary to [7, Lemma 2.4, p. 181] we have

$$
l^{-4 j}\left|R_{j}\right|=l^{3 j}+\sum_{k=0}^{j-1} l^{3 k}\left(1-l^{-1}\right)
$$

for $1 \leq j \leq \nu$. Thus our assertion follows.

Next we consider the cases $q\left(\bmod l^{\nu}\right)$ is nonzero in $\boldsymbol{o} / l^{\nu} \boldsymbol{o}$.

Lemma 1. Under the assumptions of Proposition 1, suppose that for $\nu \geq \tau(q)+1$ we have

$$
S_{\nu}=l^{-4 \nu} \sum_{\substack{p: \boldsymbol{O} / l^{\nu} \boldsymbol{O} \\ N(p) \equiv 0 \\\left(\bmod l^{\nu}\right)}} e^{2 \pi i \sigma(q, p) / l^{\nu}}
$$

Then

$$
S_{\nu}=\sum_{k=0}^{\tau(q)} l^{3 k}\left(1-l^{-1}\right)
$$

Proof. By the $p$-adic version of the Siegel-Babylonian process, we have

$$
S_{\nu}=l^{-5 \nu} \sum_{u=1}^{l^{\nu}} \sum_{p: \boldsymbol{O} / l^{\nu} \boldsymbol{O}} \exp \left\{2 \pi i[\sigma(q, p)+u N(p)] / l^{\nu}\right\} .
$$

Separate the sum according to whether $u$ is divisible by $l$ or not. Thus we have

$$
S_{\nu}=S_{\nu}(1)+S_{\nu}(2)
$$

with

$$
\begin{aligned}
S_{\nu}(1) & =l^{-5 \nu} \sum_{(u, l)=1} \sum_{p: \boldsymbol{O} / l^{\nu} \boldsymbol{O}} \exp \left\{2 \pi i[\sigma(q, p)+u N(p)] / l^{\nu}\right\} \\
& =l^{-5 \nu} \sum_{(u, l)=1} \sum_{p: \boldsymbol{O} / l^{\nu} \boldsymbol{O}} \exp \left\{2 \pi i u N\left(p+\frac{q}{u}\right) / l^{\nu}\right\} \\
& =l^{-5 \nu}\left(l^{\nu}-l^{\nu-1}\right) l^{4 \nu} \\
& =1-l^{-1}
\end{aligned}
$$


and

$$
S_{\nu}(2)=l^{-5 \nu} \sum_{u^{\prime}=1}^{l^{\nu-1}} \sum_{p: \boldsymbol{O} / l^{\nu} \boldsymbol{O}} \exp \left\{2 \pi i \sigma(q, p) / l^{\nu}+2 \pi i u^{\prime} N(p) / l^{\nu-1}\right\} .
$$

Set $p=p^{\prime}+l^{\nu-1} r$, where $p^{\prime} \in \boldsymbol{o} / l^{\nu-1} \boldsymbol{o}$ and $r \in \boldsymbol{o} / \boldsymbol{o}$ in the second summation of $S_{\nu}(2)$. Then

$$
S_{\nu}(2)=l^{-5 \nu} \sum_{u^{\prime}=1}^{l^{\nu-1}} \sum_{p^{\prime}: \boldsymbol{O} / l^{\nu-1} \boldsymbol{O}} \exp \left\{2 \pi i\left[\sigma\left(q, p^{\prime}\right) / l^{\nu}+u^{\prime} N\left(p^{\prime}\right)\right] / l^{\nu-1}\right\} \sum_{r: \boldsymbol{O} / l \boldsymbol{O}} e^{2 \pi i \sigma(q, r) / l} .
$$

Thus $S_{\nu}(2)=0$ unless $l^{-1} q \in \boldsymbol{o}$. Set $l^{-1} q=q^{\prime}$. Then

$$
S_{\nu}(2)=l^{-5 \nu+8} \sum_{u^{\prime}=1}^{l^{\nu-1}} \sum_{p^{\prime}: \boldsymbol{O} / l^{\nu-1} \boldsymbol{O}} \exp \left\{2 \pi i\left[\sigma\left(q^{\prime}, p^{\prime}\right)+u^{\prime} N\left(p^{\prime}\right)\right] / l^{\nu-1}\right\} .
$$

When we repeat the above process on $S_{\nu}$ to $S_{\nu}(2)$, we get

$$
S_{\nu}(2)=l^{3}\left(1-l^{-1}\right)+S_{\nu-2}
$$

if $\tau \geq 1$. Thus our assertion follows by an induction on $\nu$.

Proposition 2. Under the same assumptions of Proposition 1, we have

$$
\frac{1}{240} \cdot l^{-4 \nu} \sum_{p: \boldsymbol{O} / l^{\nu} \boldsymbol{O}} e^{2 \pi i \sigma(q, p) / l^{\nu}} F_{\nu}(p)=\sum_{k=0}^{\tau(q)} l^{3 k}=\frac{1}{240} \cdot F_{\nu}(q),
$$

where $\tau(q)=\tau\left(l^{\nu}, q\right)$.

Proof. By our definition of $F_{\nu}(p)$ we have

$$
\begin{aligned}
& \frac{1}{240} \cdot l^{-4 \nu} \sum_{p: \boldsymbol{O} / l^{\nu} \boldsymbol{O}} e^{2 \pi i \sigma(q, p) / l^{\nu}} F_{\nu}(p) \\
& =S_{\nu}+l^{-1} S_{\nu-1}+\ldots+l^{-(\nu-\tau-1)} S_{\tau+1}+l^{-(\nu-\tau)}\left|R_{\tau}\right|+\ldots+l^{-(\nu-1)}\left|R_{1}\right|+l^{-\nu},
\end{aligned}
$$

where $S_{j}$ is as given in Lemma 1 and $R_{j}$ is as given in Proposition 1. Our assertion then follows from the fact that

$$
S_{\nu}=S_{\nu-1}=\ldots=S_{\tau+1}=\sum_{k=0}^{\tau(q)} l^{3 k}\left(1-l^{-1}\right)
$$

and that for $1 \leq j \leq \tau(q)$ we have

$$
\left|R_{j}\right|=l^{3 j}+\sum_{k=0}^{j-1} l^{3 k}\left(1-l^{-1}\right)
$$




\section{JACOBI FORMS ON THE HERMitian UPPER PLANE OF DEGREE TWO}

In order to investigate the Fourier-Jacobi expansions of modular forms on the exceptional domain, we have to develop the theory of Jacobi forms on $\mathcal{H}_{2} \times\left(\mathcal{C}_{\mathbf{C}}\right)^{2}$.

Let $k, m$ be nonnegative integers. A holomorphic function $f: \mathcal{H}_{2} \times\left(\mathcal{C}_{\mathbf{C}}\right)^{2}$ is called a Jacobi form of weight $k$ and index $m$ if it satisfies the following conditions:

(1) $f(Z+T, W)=f(Z, W)$ for all $T=\left[\begin{array}{cc}n_{1} & t \\ \bar{t} & n_{2}\end{array}\right]$, where $n_{1}, n_{2} \in \mathbf{Z}$ and $t \in \boldsymbol{o}$;

(2) $f(Z[U], W U)=f(Z, W)$ for all $U=\left[\begin{array}{ll}1 & t \\ 0 & 1\end{array}\right]$ or $\left[\begin{array}{ll}1 & 0 \\ t & 1\end{array}\right]$ or $\left[\begin{array}{ll}0 & 1 \\ 1 & 0\end{array}\right]$, where $t \in \boldsymbol{O}$

(3) $f\left(-Z^{-1}, Z^{-1} W\right)=(\operatorname{det} Z)^{k} \exp \left\{2 \pi i m Z^{-1}[W]\right\} f(Z, W)$;

(4) $f(Z, W+Z q+p)=\exp \left\{-2 \pi i m\left(Z[q]+\sigma\left(q_{1}, w_{1}\right)+\sigma\left(q_{2}+w_{2}\right)\right)\right\} f(Z, W)$ for all $q, p \in \boldsymbol{o}^{2}$; and

(5) $f$ has a Fourier expansion of the form

$$
f(Z, W)=\sum_{q={ }^{t}\left(q_{1}, q_{2}\right) \in \boldsymbol{O}^{2}} \sum_{T \geq q^{t} \bar{q} / m} a(T, q) e^{2 \pi i\left[(Z, T)+\sigma\left(q_{1}, w_{1}\right)+\sigma\left(q_{2}, w_{2}\right)\right]} .
$$

Here for $Z=\left[\begin{array}{cc}z_{1} & z_{12} \\ \bar{z}_{12} & z_{2}\end{array}\right] \in \mathcal{H}_{2}$ and $W=\left[\begin{array}{c}w_{1} \\ w_{2}\end{array}\right] \in\left(\mathcal{C}_{\mathbf{C}}\right)^{2}$, we let

$$
Z[W]=z_{1} N\left(w_{1}\right)+z_{2} N\left(w_{2}\right)+\sigma\left(z_{12} w_{2}, w_{1}\right)
$$

Natural examples of Jacobi forms on $\mathcal{H}_{2} \times\left(\mathcal{C}_{\mathbf{C}}\right)^{2}$ come from Fourier-Jacobi expansions of modular forms on the 27-dimensional exceptional domain. Here we shall investigate the Jacobi forms corresponding to the singular modular form of weight 4 constructed by Kim in [8]. Before doing this, we shall investigate the general properties of Jacobi forms defined as above.

For each $q={ }^{t}\left(q_{1}, q_{2}\right) \in \boldsymbol{o}^{2}$, consider the theta series $\vartheta_{m, q}(Z, W)$ defined by

$$
\begin{aligned}
& \vartheta_{m, q}(Z, W) \\
& =\sum_{h=\lambda+q / m, \lambda \in \boldsymbol{O}^{2}} \exp \left\{2 \pi i m\left[\left(h^{t} \bar{h}, Z\right)+\sigma\left(h_{1}, w_{1}\right)+\sigma\left(h_{2}, w_{2}\right)\right]\right\} .
\end{aligned}
$$

Obviously, one has the identities

$$
\vartheta_{m, q}(Z+T, W)=e^{2 \pi i\left(q^{t} \bar{q}, T\right) / m} \vartheta_{m, q}(Z, W) \text { for } T=\left[\begin{array}{cc}
n_{1} & l \\
\bar{\lambda} & n_{2}
\end{array}\right] \text {, }
$$

where $n_{1}, n_{2} \in \mathbf{Z}$ and $\lambda \in \boldsymbol{o}$,

and

$$
\begin{aligned}
& \vartheta_{m, q}(Z[U], W U)=\vartheta_{m, U q}(Z, W) \text { for } U=\left[\begin{array}{cc}
0 & 1 \\
-1 & 0
\end{array}\right] \\
& \text { or } U=\left[\begin{array}{ll}
1 & t \\
0 & 1
\end{array}\right], \text { where } t \in \boldsymbol{o} .
\end{aligned}
$$

Here we shall construct the transformation formula between $\vartheta_{m, q}\left(-Z^{-1}, Z^{-1} W\right)$ and $\vartheta_{m, p}(Z, W)$. We need the following lemma.

Lemma 2. For each $h={ }^{t}\left(h_{1}, h_{2}\right) \in \mathcal{C}_{\mathbf{R}}^{2}$ and $\Lambda=\operatorname{diag}\left[\xi_{1}, \xi_{2}\right]$ where $\xi_{1}>0, \xi_{2}>0$, one has

$$
\left(h^{t} \bar{h}, \Lambda[U]\right)=\left((U h)\left({ }^{t} \bar{h}^{t} \bar{U}\right), \Lambda\right)
$$


for all

$$
U=\left[\begin{array}{ll}
1 & t \\
0 & 1
\end{array}\right],\left[\begin{array}{ll}
1 & 0 \\
t & 1
\end{array}\right] \text { or }\left[\begin{array}{ll}
0 & 1 \\
1 & 0
\end{array}\right]
$$

where $t \in \mathcal{C}_{\mathbf{R}}$.

Proof. It is obvious for $U=\left[\begin{array}{ll}0 & 1 \\ 1 & 0\end{array}\right]$. We examine the case $U=\left[\begin{array}{ll}1 & t \\ 0 & 1\end{array}\right]$. We have

$$
\left(h^{t} \bar{h}, \Lambda[U]\right)=\xi_{1} N\left(h_{1}\right)+\xi_{1} \sigma\left(t, h_{1} \bar{h}_{2}\right)+\left(\xi_{2}+\xi_{1} N(t)\right) N\left(h_{2}\right) .
$$

On the other hand, we also have

$$
U h=\left[\begin{array}{c}
h_{1}+t h_{2} \\
h_{2}
\end{array}\right] .
$$

It follows that

$$
\begin{aligned}
\left((U h)\left({ }^{t} \bar{h}^{t} \bar{U}\right), \Lambda\right) & =\xi_{1} N\left(h_{1}+t h_{2}\right)+\xi_{2} N\left(h_{2}\right) \\
& =\xi_{1} N\left(h_{1}\right)+\xi_{1} \sigma\left(h_{1}, t h_{2}\right)+\left(\xi_{1} N\left(t_{1}\right)+\xi_{2}\right) N\left(h_{2}\right) .
\end{aligned}
$$

Hence our assertion follows from the fact that

$$
\sigma\left(t, h_{1} \bar{h}_{2}\right)=T\left(t\left(h_{2} \bar{h}_{1}\right)\right)=T\left(\left(t h_{2}\right) \bar{h}_{1}\right)=\sigma\left(h_{1}, t h_{2}\right) .
$$

With the same argument we have the following lemma.

Lemma 3. For $h={ }^{t}\left(h_{1}, h_{2}\right) \in \mathcal{C}_{\mathbf{R}}^{2}, V={ }^{t}\left(v_{1}, v_{2}\right) \in \mathcal{C}_{\mathbf{C}}^{2}, \Lambda=\operatorname{diag}\left[\xi_{1}, \xi_{2}\right]$, where $\xi_{1}>0, \xi_{2}>0$, and

$$
U=\left[\begin{array}{ll}
1 & t \\
0 & 1
\end{array}\right] \text { or }\left[\begin{array}{ll}
1 & 0 \\
t & 1
\end{array}\right]
$$

where $t \in \mathcal{C}_{\mathbf{R}}$, we have

$$
T\left({ }^{t} \bar{h}\left(\Lambda^{-1}\left[{ }^{t} \bar{U}^{-1}\right]\right) V\right)=T\left(\left({ }^{t} \bar{h} U^{-1}\right)\left(\Lambda^{-1}\left({ }^{t} \bar{U}^{-1} V\right)\right)\right) .
$$

Proposition 3. For each $q=\left(q_{1}, q_{2}\right) \in \boldsymbol{o}^{2}$ we have

$$
\begin{aligned}
& \vartheta_{m, q}\left(-Z^{-1}, Z^{-1} W\right) \\
& =(\operatorname{det} Z)^{4} \exp \left\{2 \pi i m Z^{-1}[W]\right\} \\
& \quad \times \frac{1}{m^{8}} \sum_{p:(\boldsymbol{o} / m \boldsymbol{O})^{2}} \exp \left\{-2 \pi i\left[\sigma\left(q_{1}, p_{1}\right)+\sigma\left(q_{2}, p_{2}\right)\right] \vartheta_{m, p}(Z, W) .\right.
\end{aligned}
$$


Proof. It suffices to prove that (5.4) holds for $Z=i Y$ and $W=i V$. Set $Y=\Lambda[U]$ with $U=\left[\begin{array}{ll}1 & t \\ 0 & 1\end{array}\right]$ and $\Lambda=\operatorname{diag}\left[\xi_{1}, \xi_{2}\right]$. Then $Y^{-1}=\Lambda^{-1}\left[{ }^{t} U^{-1}\right]$ and

$$
\begin{aligned}
& \vartheta_{m, q}\left(i Y^{-1}, Y^{-1} V\right) \\
& =\sum_{h=\lambda+q / m, \lambda \in \boldsymbol{O}^{2}} \exp \left\{-2 \pi m\left(h^{t} \bar{h}, Y^{-1}\right)+2 \pi i T\left(\left({ }^{t} \bar{h}\right)\left(Y^{-1} V\right)\right)\right\} \\
& =\sum_{h=\lambda+q / m, \lambda \in \boldsymbol{O}^{2}} \exp \left\{-2 \pi m\left(h^{t} \bar{h}, \Lambda^{-1}\left[{ }^{t} U^{-1}\right]\right)+2 \pi i T\left({ }^{t} \bar{h}\left(\Lambda^{-1}\left[{ }^{t} \bar{U}^{-1}\right]\right) V\right)\right\} \\
& =\sum_{h=\lambda+q / m, \lambda \in \boldsymbol{O}^{2}} \exp \left\{-2 \pi m\left(\left({ }^{t} \bar{U}^{-1} h\right)\left({ }^{t} \bar{h} U^{-1}\right), \Lambda^{-1}\right)\right. \\
& \left.\quad+2 \pi i m T\left(\left({ }^{t} \bar{h} U^{-1}\right) \Lambda^{-1}\left({ }^{t} \bar{U}^{-1} V\right)\right)\right\},
\end{aligned}
$$

by Lemma 2 and Lemma 3,

$=e^{-2 \pi m \Lambda^{-1}\left[^{t} \bar{U}^{-1} V\right]}\left(\xi_{1} \xi_{2}\right)^{4} m^{-8} \sum_{h \in \boldsymbol{O}^{2}} \exp \left\{-2 \pi m^{-1}\left((U h)\left({ }^{t} \bar{h}^{t} \bar{U}\right), \Lambda\right)\right.$

$$
\left.-2 \pi T\left({ }^{t} \bar{h}^{t} \bar{U}\right)\left({ }^{t} \bar{U}^{-1}\right)\left(\frac{q}{m}-i V\right)\right\}
$$

by Poisson summation formula,

$$
\begin{aligned}
& =e^{-2 \pi m Y^{-1}[V]}(\operatorname{det} Y)^{4} m^{-8} \sum_{h \in \boldsymbol{O}^{2}} \exp \left\{-2 \pi m^{-1}\left(h^{t} \bar{h}, Y\right)\right. \\
& \left.-2 \pi i \sigma\left(\frac{q_{1}}{m}-i v_{1}, h_{1}\right)-2 \pi i \sigma\left(\frac{q_{2}}{m}-i v_{2}, h_{2}\right)\right\} \\
& =e^{-2 \pi m Y^{-1}[V]}(\operatorname{det} Y)^{4} m^{-8} \sum_{p:(\boldsymbol{O} / m \boldsymbol{O})^{2}} \exp \left\{-2 \pi i\left[\sigma\left(q_{1}, p_{1}\right)+\sigma\left(q_{2}, p_{2}\right)\right] / m\right\} \\
& \times \sum_{h=\lambda+p / m, \lambda \in \boldsymbol{O}^{2}} \exp \left\{-2 \pi m\left(h^{t} \bar{h}, Y\right)-2 \pi m\left(\sigma\left(v_{1}, h_{1}\right)+\sigma\left(v_{2}, h_{2}\right)\right\}\right. \\
& =e^{-2 \pi m Y^{-1}[V]}(\operatorname{det} Y)^{4} m^{-8} \sum_{p:(\boldsymbol{O} / m \boldsymbol{O})^{2}} \exp \left\{-2 \pi i\left[\sigma\left(q_{1}, p_{1}\right)\right.\right. \\
& \left.\left.+\sigma\left(q_{2}, p_{2}\right)\right] / m\right\} \vartheta_{m, p}(i Y, i V) .
\end{aligned}
$$

This proves our assertion.

Let $\Gamma_{2}$ be the arithmetic group of the group of automorphisms of $\mathcal{H}_{2}$ generated by

(A) $p_{B}: Z \longrightarrow Z+B$, for $B=\left[\begin{array}{cc}n_{1} & t \\ \bar{t} & n_{2}\end{array}\right]$ where $n_{1}, n_{2} \in \mathbf{Z}$ and $t \in \boldsymbol{o}$,

(B) $\iota: Z \longrightarrow-Z^{-1}$, and

(C) $t_{U}: Z \longrightarrow Z[U]$, for $U=\left[\begin{array}{cc}0 & 1 \\ -1 & 0\end{array}\right]$ or $U=\left[\begin{array}{ll}1 & t \\ 0 & 1\end{array}\right]$ where $t \in \boldsymbol{o}$.

As Jacobi forms on $\mathcal{H}_{1} \times \mathcal{C}_{\mathbf{C}}$, we have the following result.

Proposition 4. There exists a group homomorphism $\psi: \Gamma_{2} \longrightarrow U\left(m^{16}\right)$, the unitary group of size $m^{16}$, determined by

(A) $\psi\left(p_{B}\right)=\operatorname{diag}\left[e^{-2 \pi i\left(q^{t} \bar{q}, B\right) / m}\right]_{q:(\boldsymbol{O} / m \boldsymbol{O})^{2}}$,

(B) $\psi(\iota)=\frac{1}{m^{8}}\left[e^{2 \pi i\left[\sigma\left(q_{1}, p_{1}\right)+\sigma\left(q_{2}, p_{2}\right)\right] / m}\right]_{p, q:(\boldsymbol{O} / m \boldsymbol{O})^{2}}$, and 
(C) $\psi\left(t_{U}\right)=\left[s_{p, q}\right]_{p, q:(\boldsymbol{O} / m \boldsymbol{O})^{2}}$, where

$s_{p, q}=\left\{\begin{array}{ll}1, & \text { if } q=U p, \\ 0, & \text { otherwise, }\end{array} \quad\right.$ for $U=\left[\begin{array}{cc}0 & 1 \\ -1 & 0\end{array}\right]$ or $U=\left[\begin{array}{ll}1 & t \\ 0 & 1\end{array}\right]$ where $t \in \boldsymbol{o}$.

Remark. Fix a set of representatives $q_{1}, \ldots, q_{m^{8}}$ of $\boldsymbol{o} / m \boldsymbol{o}$ and let

$$
A=\frac{1}{m^{4}}\left[e^{2 \pi i \sigma\left(q_{\mu}, q_{\nu}\right) / m}\right]_{1 \leq \mu, \nu \leq m^{8}} .
$$

It is easy to see that

$$
\psi(\iota)=A \otimes A,
$$

where

$$
M \otimes N=\left[\begin{array}{cccc}
m_{11} N & m_{12} N & \ldots & m_{1 n} N \\
m_{21} N & m_{22} N & \ldots & m_{2 n} N \\
\vdots & \vdots & & \vdots \\
m_{p 1} N & m_{p 2} N & \ldots & m_{p n} N
\end{array}\right]
$$

if $M=\left[m_{i j}\right]_{1 \leq i \leq p, 1 \leq j \leq n}$.

\section{THE SECOND IDENTITY}

For each positive integer $m$ and $q={ }^{t}\left(q_{1}, q_{2}\right) \in \boldsymbol{o}^{2}$ with $q^{t} \bar{q} \equiv 0(\bmod m)$, we define

$$
T(m, q)=\left[\begin{array}{cc}
q^{t} \bar{q} / m & q \\
{ }^{t} \bar{q} & m
\end{array}\right]=\left[\begin{array}{ccc}
N\left(q_{1}\right) / m & q_{1} \bar{q}_{2} / m & q_{1} \\
q_{2} \bar{q}_{1} / m & N\left(q_{2}\right) / m & q_{2} \\
\bar{q}_{1} & \bar{q}_{2} & m
\end{array}\right] .
$$

Obviously, we have $T(m, q) \in \mathcal{J}_{\boldsymbol{o}}$ and $\operatorname{rank} T(m, q)=1$. Let

$$
d(m, q)=\max \left\{\alpha \in \mathbf{N} \mid \alpha^{-1} T(m, q) \in \mathcal{J}_{\boldsymbol{o}}\right\} .
$$

Now define

$$
G_{m}(q)=\left\{\begin{array}{cl}
240 \sum_{k \mid d(m, q)} k^{3}, & \text { if } q^{t} \bar{q} \equiv 0 \quad(\bmod m), \\
0, & \text { otherwise }
\end{array}\right.
$$

We shall prove the identity

$$
G_{m}(q)=m^{-8} \sum_{p \in(\boldsymbol{O} / m \boldsymbol{O})^{2}} e^{2 \pi i\left[\sigma\left(q_{1}, p_{1}\right)+\sigma\left(q_{2}, p_{2}\right)\right] / m} G_{m}(p) .
$$

Since the functions in both sides of the equality are multiplicative functions of $m$, it suffices to consider the cases $m=l^{\nu}$ for some prime number $l$ and positive integer $\nu$. We write $T(\nu, q), d(\nu, q), G_{\nu}(q)$ to replace $T\left(l^{\nu}, q\right), d\left(l^{\nu}, q\right), G_{l^{\nu}}(q)$ respectively.

First we consider the case $q=0$. We need the following useful tools from [7].

Lemma 4. Let $l$ be a prime number, $\nu$ be a positive integer and $x$ be a primitive element of $\boldsymbol{o} / l^{\nu} \boldsymbol{o}$ with $N(x) \equiv 0\left(\bmod l^{\nu}\right)$. Suppose that $y \in \boldsymbol{o}$. Then $\bar{x} y \equiv 0$ $\left(\bmod l^{\nu}\right)$ if and only if $y=x z\left(\bmod l^{\nu}\right)$. As $z$ varies in $\boldsymbol{o} / l^{\nu} \boldsymbol{o}$, there are $l^{4 \nu}$ such $y\left(\bmod l^{\nu}\right)$ and each $y\left(\bmod l^{\nu}\right)$ is assumed $l^{4 \nu}$ times.

Lemma 5. The number of elements $x$ in $\boldsymbol{o} / l^{\nu} \boldsymbol{o}$ such that $N(x) \equiv 0\left(\bmod l^{\nu}\right)$ is given by

$$
l^{4 \nu}\left\{l^{3 \nu}+\sum_{k=0}^{\nu-1} l^{3 k}\left(1-l^{-1}\right)\right\} .
$$


Lemma 6. The number of primitive elements $x$ in $\boldsymbol{o} / l^{\nu} \boldsymbol{o}$ with $N(x) \equiv 0\left(\bmod l^{\nu}\right)$ is given by $\left(l^{7 \nu}+l^{7 \nu-3}\right)\left(1-l^{-4}\right)$.

Proof. Let $A_{\nu}$ be the number of elements $x$ is $\boldsymbol{o} / l^{\nu} \boldsymbol{o}$ such that $N(x) \equiv 0\left(\bmod l^{\nu}\right)$. Then the number of primitive elements in $A_{\nu}$ is

$$
A_{\nu}-l^{8} A_{\nu-2}=\left(l^{7 \nu}+l^{7 \nu-3}\right)\left(1-l^{-4}\right) .
$$

Now we are ready to prove that the $l$-adic version of identity (II) holds for $q=0$.

Proposition 5. Let $l$ be a prime number and $\nu$ be a nonnegative integer. Then

$$
\frac{1}{240} \cdot G_{\nu}(0)=\frac{1}{240} \cdot l^{-8 \nu} \sum_{p \in\left(\boldsymbol{O} / l^{\nu} \boldsymbol{O}\right)^{2}} G_{\nu}(p)=\sum_{k=0}^{\nu} l^{3 k} .
$$

Proof. For integers $j$ with $0 \leq j \leq \nu$ we have

$$
R_{j}=\left\{T(j, q) \mid q \in\left(\boldsymbol{o} / l^{\nu} \boldsymbol{o}\right)^{2}, q^{t} \bar{q} \equiv 0 \quad\left(\bmod l^{j}\right)\right\} .
$$

Note that if $T(j, q) \in R_{j}$ and $l^{-k} T(j, q) \in \mathcal{J}_{\boldsymbol{o}_{l}}, 0 \leq k \leq j$, then $l^{-k} T(j, q)=$ $T\left(j-k, l^{-k} q\right) \in R_{j-k}$. Hence, by our definition we have

$$
\begin{aligned}
& \frac{1}{240} \cdot l^{-8 \nu} \sum_{p \in\left(\boldsymbol{O} / l^{\nu} \boldsymbol{O}\right)^{2}} G_{\nu}(p) \\
& =l^{-8 \nu}\left|R_{\nu}\right|+l^{-8 \nu+3}\left|R_{\nu-1}\right|+\ldots+l^{-8 \nu+3(\nu-1)}\left|R_{1}\right|+l^{-8 \nu+5 \nu},
\end{aligned}
$$

where $\left|R_{j}\right|$ denotes the number of matrices in $R_{j}$.

Our assertion will follow if we can prove that

$$
l^{-8 j}\left|R_{j}\right|=l^{3 j}+\sum_{k=0}^{j-1} l^{3 k}\left(1-l^{-5}\right), \quad 1 \leq j \leq \nu .
$$

Note that

$$
\left|R_{j}\right|=\left|\left\{q \in\left(\boldsymbol{o} / l^{j} \boldsymbol{o}\right)^{2} \mid q^{t} \bar{q} \equiv 0 \quad\left(\bmod l^{j}\right)\right\}\right| .
$$

Set $q={ }^{t}\left(q_{1}, q_{2}\right)$. Then $q^{t} \bar{q} \equiv 0\left(\bmod l^{j}\right)$ if and only if

$$
N\left(q_{1}\right) \equiv q_{1} \bar{q}_{2} \equiv N\left(q_{2}\right) \equiv 0 \quad\left(\bmod l^{j}\right) .
$$

Let $S_{j}$ (respectively $T_{j}$ ) be the subset of $R_{j}$ corresponding to the cases $q_{1}$ is primitive (respectively $q_{1}$ is primitive and $q_{2}$ is nonprimitive). By Lemma 4 and Lemma 6, we have

$$
\left|S_{j}\right|=\left(l^{7 \nu}+l^{7 \nu-3}\right)\left(1-l^{-4}\right) l^{4 \nu}
$$

and

$$
\left|T_{j}\right|=\left(l^{7 \nu}+l^{7 \nu-3}\right)\left(1-l^{-4}\right) l^{4(\nu-1)} .
$$

Consequently, we have

$$
\begin{aligned}
l^{-8 j}\left|R_{j}\right| & =l^{-8 j}\left\{\left|S_{j}\right|+\left|T_{j}\right|\right\}+l^{-8(j-2)}\left|R_{j-2}\right| \\
& =\left(l^{3 j}+l^{3(j-1)}\right)\left(1-l^{-8}\right)+l^{-8(j-2)}\left|R_{j-2}\right|
\end{aligned}
$$

for $j \geq 2$. Thus our assertion follows from the fact $\left|R_{0}\right|=1$, the equality

$$
l^{-8}\left|R_{1}\right|=l^{3}+\left(1-l^{-5}\right)
$$

and induction on $j$. 
Next we consider the general case. Set $\tau(q)=\tau(\nu, q)=\nu_{l}(d(\nu, q))$. In other words, $l^{\tau(q)}$ is the largest exponent of $l$ such that $\tau(q) \leq \nu$ and $l^{-\tau(q)} T(\nu, q) \in \mathcal{J}_{\boldsymbol{o}_{l}}$. With such notation, we can rewrite $G(\nu, q)$ as

$$
G_{\nu}(q)=240 \sum_{k=0}^{\tau(q)} l^{k}
$$

We need the following lemmas from 7 .

Lemma 7. Let $l$ be a prime number and $\nu$ be a positive integer. Suppose that $q \in \boldsymbol{o} / l^{\nu} \boldsymbol{o}$ with $N(q) \equiv 0\left(\bmod l^{\nu}\right)$ and $A_{\nu}(q)$ is the number of $y\left(\bmod l^{\nu}\right)$ such that $\bar{q} y \equiv N(y) \equiv 0\left(\bmod l^{\nu}\right)$. Then

$$
A_{\nu}(q)=l^{4 \nu}\left\{l^{3 \tau(q)}+\sum_{k=0}^{\tau(q)-1} l^{3 k}\left(1-l^{-1}\right)\right\},
$$

where $\tau(q)=\tau(\nu, q)$.

Lemma 8. Under the same assumption of the previous lemma. Let $B_{\nu}(q)$ be the number of primitive elements $y\left(\bmod l^{\nu}\right)$ such that $\bar{q} y \equiv N(y) \equiv 0\left(\bmod l^{\nu}\right)$. Then

$$
B_{\nu}(q)=l^{4 \nu+3 \tau(q)}\left(1-l^{-4}\right) .
$$

Proof. It follows from the fact

$$
B_{\nu}(q)=A_{\nu}(q)-l^{8} A_{\nu-2}\left(l^{-1} q\right) \text { if } \tau \geq 1 .
$$

Note that $G_{\nu}(q)$ is invariant under the transformation $q \longrightarrow U q$ with $U=$ $\left[\begin{array}{ll}1 & 0 \\ t & 1\end{array}\right],\left[\begin{array}{ll}1 & t \\ 0 & 1\end{array}\right]$ where $t \in \boldsymbol{o}$, or $U=\left[\begin{array}{ll}0 & 1 \\ 1 & 0\end{array}\right]$. The sum

$$
\begin{aligned}
& l^{-8 \nu} \sum_{p \in\left(\boldsymbol{O} / l^{\nu} \boldsymbol{O}\right)^{2}} e^{2 \pi i\left[\left(q_{1}, p_{1}\right)+\left(q_{2}, p_{2}\right)\right] / l^{\nu}} G_{\nu}(p) \\
& =l^{-8 \nu} \sum_{p:\left(\boldsymbol{O} / l^{\nu} \boldsymbol{O}\right)^{2}} e^{2 \pi i T\left({ }^{t} \bar{q} p\right) / l^{\nu}} G_{\nu}(p)
\end{aligned}
$$

is invariant under the transformation $q \longrightarrow U q$ as well. On the other hand, for each $x \in \mathcal{C}_{\mathbf{R}}$, there exists $c \in \boldsymbol{o}$ such that $N(x-c) \leq \frac{1}{2}$ (see [1]). Thus the usual Euclidean algorithm for integers can be extended to integral Cayley numbers. Without loss of generality, we can assume the second component $q_{2}$ of $q$ is zero.

Proposition 6. Let $l$ be a prime number and $\nu$ be a positive integer such that $\nu>\tau=\tau\left(l^{\nu}, q_{1}\right)$. Then

$$
\frac{1}{240} l^{-8 \nu} \sum_{p \in\left(\boldsymbol{O} / l^{\nu} \boldsymbol{O}\right)^{2}} e^{2 \pi i \sigma\left(q_{1}, p_{1}\right) / l^{\nu}} G_{\nu}(p)=\sum_{k=0}^{\tau} l^{3 k}=\frac{1}{240} \cdot G_{\nu}\left(\left(\begin{array}{c}
q_{1} \\
0
\end{array}\right)\right) .
$$

Proof. For integers $j$ with $\tau+1 \leq j \leq \nu$, let

$$
S_{j}=l^{-8 j} \sum_{\substack{p \in\left(\boldsymbol{O} / l^{\nu} \boldsymbol{O}\right)^{2} \\ p^{t} \bar{p} \equiv 0 \\(\bmod l j)}} e^{2 \pi i\left(q_{1}, p_{1}\right) / l^{\nu}},
$$


and $R_{j}$ be as defined in Proposition 5. Then we have

$$
\begin{aligned}
& \frac{1}{240} \cdot l^{-8 \nu} \sum_{p \in\left(\boldsymbol{O} / l^{\nu} \boldsymbol{O}\right)^{2}} e^{2 \pi i \sigma\left(q_{1}, p_{1}\right) / l^{\nu}} G_{\nu}(p)=S_{\nu}+l^{-5} S_{\nu-1}+\ldots+l^{-5(\nu-\tau-1)} S_{\tau+1} \\
& \quad+l^{-5(\nu-\tau)}\left|R_{\tau}\right|+\ldots+l^{-5(\nu-1)}\left|R_{1}\right|+l^{-5 \nu} .
\end{aligned}
$$

Thus the equality holds if we can prove that

$$
S_{j}=\sum_{k=0}^{\tau} l^{3 k}\left(1-l^{-5}\right)
$$

for $\tau+1 \leq j \leq \nu$. Consider the following partial sums of $S_{j}$ :

(1) $S_{j}(1)$ : sum over all $p={ }^{t}\left(p_{1}, p_{2}\right)$ where $p_{1}$ is primitive,

(2) $S_{j}(2)$ : sum over all $p={ }^{t}\left(p_{1}, p_{2}\right)$ where both $p_{1}, p_{2}$ are primitive,

(3) $S_{j}(3)$ : sum over all $p={ }^{t}\left(p_{1}, p_{2}\right)$ where $p_{2}$ is primitive, and

(4) $S_{j}(4)$ : sum over all $p={ }^{t}\left(p_{1}, p_{2}\right)$ where $p_{1}, p_{2}$ are non-primitive.

Obviously, we have

$$
S_{j}=S_{j}(1)-S_{j}(2)+S_{j}(3)+S_{j}(4) .
$$

If $j \geq \tau+2$, we have

$$
S_{j}(4)=S_{j-2} \text {. }
$$

By Lemma 4, we have

$$
\begin{aligned}
S_{j}(1) & =l^{-4 j} \sum_{\substack{p_{1} \in \boldsymbol{O} / l^{j} \boldsymbol{O} \\
p_{1} \text { primitive } \\
N\left(p_{1}\right) \equiv 0 \\
\left(\bmod l^{j}\right)}} e^{2 \pi i \sigma\left(q_{1}, p_{1}\right) / l^{j}} \\
& =l^{-4 j} \sum_{\substack{\left.N\left(p_{1}\right) \equiv 0 \bmod l^{j}\right) \\
p_{1} \in \boldsymbol{O} / l^{j} \boldsymbol{O}}} e^{2 \pi i \sigma\left(q_{1}, p_{1}\right) / l^{j}}-l^{-4(j-2)} \sum_{\substack{N\left(p^{\prime}\right) \equiv 0\left(\bmod l^{j-2}\right) \\
p^{\prime} \in \boldsymbol{O} / l^{j-2} \boldsymbol{O}}} e^{2 \pi i \sigma\left(q_{1}, p^{\prime}\right) / l^{j-1}} \\
& =l^{3 \tau}\left(1-l^{-4}\right), \quad \text { by Lemma 1. }
\end{aligned}
$$

It follows easily that

$$
S_{j}(2)=l^{3 \tau}\left(1-l^{-1}\right)\left(1-l^{-4}\right) .
$$

Again by Lemma 4 we have

$$
\begin{aligned}
S_{j}(3) & =l^{-8 j} \sum_{\substack{p_{2} \in \boldsymbol{O} / l^{j} \boldsymbol{O} \\
p_{2} \text { primitive } \\
N\left(p_{2}\right) \equiv 0\left(\bmod l^{j}\right)}} l^{-4 j} \sum_{z \in \boldsymbol{O} / l^{j} \boldsymbol{O}} e^{2 \pi i \sigma\left(q_{1}, p_{2} z\right)} \\
& =l^{-4 j} \mid\left\{p_{2} \in \boldsymbol{o} / l^{j} \boldsymbol{o} \mid p_{2} \text { is primitive and } \bar{p}_{2} q \equiv 0 \quad\left(\bmod l^{j}\right)\right\} \mid \\
& =l^{3 \tau}\left(1-l^{-4}\right), \quad \text { by Lemma } 8 .
\end{aligned}
$$

Consequently, we have

$$
S_{j}(1)-S_{j}(2)+S_{j}(3)=l^{3 \tau}\left(1-l^{-5}\right) .
$$

By the same process as above we are able to show that

$$
S_{\tau+1}=\sum_{k=0}^{\tau} l^{3 k}\left(1-l^{-5}\right)
$$

Thus our assertion for $S_{j}$ follows by induction on $j$. 


\section{A CONSEQUence OF THE SECOND IDENTity}

Here we combine the theory of Section 5 with the second identity to yield a Jacobi form of weight 4 and index $m$ on $\mathcal{H}_{2} \times\left(\mathcal{C}_{\mathbf{C}}\right)^{2}$.

Proposition 7. With the theta series $\vartheta_{m, q}(Z, W)$ defined in (5.1) and the constant $G_{m}(q)$ defined in (6.2), the function

$$
\varphi_{m}(Z, W)=\sum_{q:(\boldsymbol{O} / m \boldsymbol{O})^{2}} G_{m}(q) \vartheta_{m, q}(Z, W)
$$

is a Jacobi form of weight 4 and index $m$ on $\mathcal{H}_{2} \times\left(\mathcal{C}_{\mathbf{C}}\right)^{2}$.

Proof. If we combine identity (II) with Proposition 3 , we get

$$
\begin{aligned}
\varphi_{m} & \left(-Z^{-1}, Z^{-1} W\right) \\
= & \sum_{q:(\boldsymbol{O} / m \boldsymbol{O})^{2}} G_{m}(q) \vartheta_{m, q}\left(-Z^{-1}, Z^{-1} W\right) \\
= & (\operatorname{det} Z)^{4} \exp \left\{2 \pi i m Z^{-1}[W]\right\} \\
& \times \sum_{p \in(\boldsymbol{O} / m \boldsymbol{O})^{2}} G_{m}(q) \frac{1}{m^{8}} \sum_{p:(\boldsymbol{O} / m \boldsymbol{O})^{2}} e^{-2 \pi i\left[\sigma\left(q_{1}, p_{1}\right)+\sigma\left(q_{2}, p_{2}\right)\right] / m} \vartheta_{m, p}(Z, W) \\
= & (\operatorname{det} Z)^{4} \exp \left\{2 \pi i m Z^{-1}[W]\right\} \\
& \times \sum_{p \in(\boldsymbol{O} / m \boldsymbol{O})^{2}} \frac{1}{m^{8}} \sum_{q: \boldsymbol{O} / m \boldsymbol{O}} e^{-2 \pi i\left[\sigma\left(q_{1}, p_{1}\right)+\sigma\left(q_{2}, p_{2}\right)\right] / m} G_{m}(q) \cdot \vartheta_{m, p}(Z, W) \\
= & (\operatorname{det} Z)^{4} \exp \left\{2 \pi i m Z^{-1}[W]\right\} \sum_{p \in(\boldsymbol{O} / m \boldsymbol{O})^{2}} G_{m}(p) \vartheta_{m, p}(Z, W) \\
= & (\operatorname{det} Z)^{4} \exp \left\{2 \pi i m Z^{-1}[W]\right\} \varphi_{m}(Z, W) .
\end{aligned}
$$

The remaining conditions are easy to verify directly, so we omit the proofs here.

Theorem. Set $Z_{1}=\left[\begin{array}{cc}z_{1} & z_{12} \\ z_{12} & z_{2}\end{array}\right], W=\left[\begin{array}{l}z_{13} \\ z_{23}\end{array}\right]$ and $Z=\left[\begin{array}{cc}Z_{1} & W \\ { }^{t} \bar{W} & z_{3}\end{array}\right]$. Then the function

$$
E(Z)=\sum_{h \in \boldsymbol{O}^{2}} e^{2 \pi i\left(h^{t} \bar{h}, Z_{1}\right)}+\sum_{m=1}^{\infty}\left(\sum_{q:(\boldsymbol{O} / m \boldsymbol{O})^{2}} G_{m}(q) \vartheta_{m, q}\left(Z_{1}, W\right)\right) e^{2 \pi i m z_{3}},
$$

where $Z \in \mathcal{H}$, is a singular modular form of weight 4 .

Proof. It is easy to see that $E(Z+B)=E(Z)$ for all $B \in \mathcal{J}_{\boldsymbol{o}}$ and for $U=U_{i j}(t)$ where $t \in \boldsymbol{o}$. We have $E(Z[U])=E(Z)$ since the coefficient $G_{m}(q)$ depends on the matrix

$$
T_{m}(q)=\left[\begin{array}{cc}
q^{t} \bar{q} / m & q \\
{ }^{t} \bar{q} & m
\end{array}\right]
$$

and so is invariant under the transformation $T_{m}(q) \longrightarrow T_{m}(q)[U]$.

Let $\iota_{12}$ be the mapping from $\mathcal{H}$ to $\mathcal{H}$ defined by

$$
\iota_{12}:\left[Z_{1}, W, z_{3}\right] \longrightarrow\left[-Z_{1}^{-1}, Z_{1}^{-1} W, z_{3}-Z_{1}^{-1}[W]\right] .
$$

By Proposition 7 we have

$$
E\left(\iota_{12}(Z)\right)=\left(\operatorname{det} Z_{1}\right)^{4} E(Z) .
$$


The detailed proofs here for the formula (7.2) and for the formula (7.6) below are the essential steps missing in Krieg's assertions (12) and (14) in his paper [9].

Rewrite $E(Z)$ as

$$
E(Z)=\sum_{R} e^{2 \pi i\left(R, Z_{1}\right)} F_{R}\left(z_{3}, z_{13}, z_{23}\right)
$$

where $R$ ranges over $2 \times 2$ Hermitian matrices over integral Cayley numbers of rank $\leq 1$ and $R \geq 0$. Indeed we have

$$
F_{R}\left(z_{3}, z_{13}, z_{23}\right)=\sum_{n R=q^{t} \bar{q}} \sum_{q \in \boldsymbol{O}^{2}} G_{m}(q) e^{2 \pi i\left[\sigma\left(q_{1}, z_{13}\right)+\sigma\left(q_{2}, z_{23}\right)\right]} e^{2 \pi i n z_{3}} .
$$

In particular, for $R=\left[\begin{array}{cc}m & 0 \\ 0 & 0\end{array}\right]$ we have

$$
\begin{aligned}
& F_{R}\left(z_{3}, z_{13}, z_{23}\right) \\
& =\sum_{q_{1} \in \boldsymbol{O}} F_{m}\left(q_{1}\right) e^{2 \pi i\left[\sigma\left(q_{1}, z_{13}\right)+z_{3} N\left(q_{1}\right) / m\right]} \\
& =\sum_{q \in \boldsymbol{O} / m \boldsymbol{O}} F_{m}(q) \sum_{l \in \boldsymbol{O}} \exp \left\{2 \pi i m N\left(l+\frac{q}{m}\right) z_{3}+\sigma\left(l+\frac{q}{m}, z_{13}\right)\right\} .
\end{aligned}
$$

By identity (I) and the transformation of the theta series in (3.1), we get

$$
F_{R}\left(-\frac{1}{z_{3}}, \frac{z_{13}}{z_{3}}, \frac{z_{23}}{z_{3}}\right)=\left(z_{3}\right)^{4} e^{2 \pi i m N\left(q_{1}\right) / z_{3}} F_{R}\left(z_{3}, z_{13}, z_{23}\right) .
$$

Under the transformation $R \longrightarrow R[U]$ with $U=\left[\begin{array}{ll}0 & 1 \\ 1 & 0\end{array}\right],\left[\begin{array}{ll}1 & 0 \\ t & 1\end{array}\right]$ or $\left[\begin{array}{ll}1 & 0 \\ t & 1\end{array}\right]$, where $t \in \boldsymbol{o}$, it causes simple transformations on $z_{13}$ and $z_{23}$. Indeed, by (17.3), we have

$$
F_{R[U]}\left(z_{3}, z_{13}^{\prime}, z_{23}^{\prime}\right)=F_{R}\left(z_{3}, z_{13}, z_{23}\right)
$$

where

$$
\left[\begin{array}{c}
z_{13}^{\prime} \\
z_{23}^{\prime}
\end{array}\right]=\bar{U}^{-1}\left[\begin{array}{l}
z_{13} \\
z_{23}
\end{array}\right] .
$$

Thus the equality in (7.4) leads to the general form

$$
F_{R}\left(-\frac{1}{z_{3}}, \frac{z_{13}}{z_{3}}, \frac{z_{23}}{z_{3}}\right)=\left(z_{3}\right)^{4} e^{2 \pi i\left(R, W^{t} \bar{W}\right) / z_{3}} F_{R}\left(z_{3}, z_{13}, z_{23}\right),
$$

where

$$
W=\left[\begin{array}{c}
z_{13} \\
z_{23}
\end{array}\right] \text { and } W^{t} \bar{W}=\left[\begin{array}{cc}
N\left(z_{13}\right) & z_{13} z_{23}^{-} \\
z_{23} z_{13}^{-} & N\left(z_{23}\right)
\end{array}\right] .
$$

Let $\iota_{3}$ be the mapping from $\mathcal{H}$ to $\mathcal{H}$ defined by

$$
\iota_{3}:\left(Z_{1}, W, z_{3}\right) \longmapsto\left(Z_{1}-\frac{1}{z_{3}} W^{t} \bar{W}, \frac{1}{z_{3}} W,-\frac{1}{z_{3}}\right) .
$$

By (7.5) we have

$$
E\left(\iota_{3}(Z)\right)=z_{3}^{4} E(Z)
$$


Note that $\iota: Z \longrightarrow-Z^{-1}$ is the composition of $\iota_{12}$ and $\iota_{3}$. By (7.2) and (7.6), we then have

$$
E\left(-Z^{-1}\right)=(\operatorname{det} Z)^{4} E(Z) .
$$

Hence $E(Z)$ is indeed a modular form of weight 4 .

Remark. The identity (7.4) is proved in 9 using the Fourier-Jacobi expansion of $f_{4}$. We wish to stress here that our proof of $(7.2)$ provides all necessary details which are practically absent in [9] and is integral to our development of the theory of Jacobi forms on $\mathcal{H}_{2} \times\left(\mathcal{C}_{\mathbf{c}}\right)^{2}$. Here we have the precise expression for $F_{R}$ and derive its transformation formula (7.3) using identity (I) and the transformation formula of the theta series.

\section{REFERENCES}

[1] W.L. Baily, Jr., An exceptional arithmetic group and its Eisenstein series, Ann. of Math. 91 (1970), 512-549. MR 42:4674

[2] W.L. Baily, Jr., Introductory lectures on automorphic forms, Princeton University Press, 1973. MR 51:5982

[3] Minking Eie, The Maass space for Cayley numbers, Math. Zeitschrift 207 (1991), 645-655. MR 92k: 11053

[4] Minking Eie and A. Krieg, The Maass space on the half-plane of Cayley numbers of degree two, Math. Zeitschrift 210 (1992), 113-128. MR 93e:11063

[5] (1994), 793-805. MR 95b:11048

[6] E. Freitag, Siegelsche Modulfunktionen, Grundlehren der mathematischen Wissenschaften 254, Springer-Verlag, Berlin, Heidelberg, New York, 1983. MR 88b:11027

[7] M. Karel, Fourier coefficients of certain Eisenstein series, Ann. of Math. 99 (1974), 176-202. MR 49:8935

[8] H. Kim, Exceptional modular form of weight 4 on an exceptional domain contained in $\mathbf{C}^{27}$, Revista Mate. Iberoam. 9 (1993), 139-200. MR 94c:11040

[9] A. Krieg, The singular modular forms on the 27-dimensional exceptional domain, Manuscripta Mathematica 92 (1997), 361-367. MR 97m:11064

[10] J.G.M. Mars, Les nombres de Tamagawa de certains groupers exceptionnels, Bull. Soc. Math. France 94 (1966), 97-140. MR 35:4227

[11] C.L. Siegel, Einführung in die Theorie der Modulfunktionen $n$-ten Grades, Math. Ann. 116 (1939), 617-657. MR 1:203f

Department of Mathematics, National Chung Cheng University, Min-Hsiung Chiayi 621, TAIWAN

E-mail address: stchang@math.ccu.edu.tw

Department of Mathematics, National Chung Cheng University, Min-Hsiung Chiayi 621, TAIWAN

E-mail address: eie@math.ccu.edu.tw 